\title{
Author Index to Volume 22
}

\author{
Abe K, 201 \\ Agsteribbe E, 394 \\ Ailabouni $\wedge, 720$ \\ Aksclrod S, 158 \\ Alden BM, 465 \\ Alvarez S, 277 \\ Anday $\mathrm{L}, 302$ \\ Anderson DC.659 \\ Andjuo O, 145 \\ Arab N, 173 \\ Araki $\wedge .500$ \\ Armstead W, 647 \\ Aronovitz $\mathrm{MJ}, 693$ \\ Artman M, 524 \\ Ashkenazi S, 130 \\ Ashwal S. 192 \\ Attanasio $\wedge, 41$ \\ Auricchio $S, 703$ \\ Bachmann C. 364 \\ Baker CJ. 698 \\ Baker SB, 471 \\ Balmer SF, 651 \\ Bancalari $\wedge .478$ \\ Bancalari 1:478 \\ Barrada $A, 16$ \\ Barratt ГM, 197 \\ Bartolotta I: 141 \\ Battaglia FC, 688 \\ Baumann (;, 2] \\ Behm JK, 271 \\ Bekedam I)J. 655 \\ Bell AW, 688 \\ Bell JM, 599 \\ Bender JG; 383 \\ Benson DW Jr, 442 \\ Berger R, 394 \\ Bergstrom WH, 414 \\ Berlin CM, 312 \\ Bernasconis 41 \\ Berner MI: 714 \\ Bernstein I), 389 \\ Bersch N, 286 \\ Bertoli I: 141 \\ Bevers F:M, 725 \\ Bhat R, 16 \\ Biol MC. 250) \\ Bland RD, 714 \\ Block (F, 150 \\ Blumer JI, 360 \\ Boucek RJ Jr, 524 \\ Brann BS IV, 135 \\ Braun I), 714 \\ Bridges RB, 736 \\ Brown $\mathrm{Cl}$ : 545 \\ Bruce I:N, 87 \\ Bryan H, 154 \\ Buchholy. RTF: 725 \\ Busija I), 647 \\ Buts J-P, 29 \\ (able I) 438 \\ Cabral D)A. 429 \\ Caces R, 344 \\ Campbell I), 324 \\ Carlo WA, 683,87 \\ Carlson KS, 79 \\ Carlson SE, 292 \\ Cashore WJ, 135 \\ Cates D, 344
}

Chance B, 302

(han JCM 518

Charlton V. 55

Chartier M. 39

Chesney RW, 755, 163

(ho) $\mathrm{BH}, 262$

Chou JW. 324

Christensen RI) 455

Chung H-T, 262

Citak M, 524

Clark EB, 442

(layton PE: 40)

Clyman RI, 714, 350, 531

Coccani $\mathrm{F}: 422$

Cochran WJ 417

Coc JY, 422

Cohen IMN, 655

Collet S, 67

Colombo JP, 364

Colton I) (i. 34

Comer M, 609

Conover (A. 188

Coonrod JD, 736

Corthier (i, 72

Curatola (;, 141

1)affos $\mathrm{F}, 39$

Danes P. 693

Darling P, 67

Dassin R. 154

Davies P. 79

Daynes RA, 262

D) Costa M, 154

Dearden LC. (9)

De Keyser : N, 29

de I ange $G .708$

Delivoria-Papadopoulos M. $3(22$

deMello DE: 693

D'ircole J, 245

Desjeux J-F. 72

de Ritis (j, 703

De Vincenzi M, 703

De Vries H, 394

DiFiore JM 683

Dimmick JI: 743

Dive (. 29)

Dobrozsi DJ. 115

Dolan I.M. 115

Dotti M, 141

Draye J-P. 748

Drever WJ, 667

Duće P-H. 587

Dunnigan $\wedge .442$

I:ckhert. (D). 268

Edwards MS. 698

Fisenfeld I, 150

Indo F. 627

Fingelhardt $\mathrm{F}: \mathrm{I}, 330$

Evans M. 16

Fairweather-Tait SJ. 651

Ferretti $G, 141$

Fewell JF: 47

Fiascone JM. 730

Finlay TH. 1

Finley S1. 432

Fiorotto ML. 417

Fisher DJ. 667

Florman AL. 383
Foreman JW, 605

Forestier F. 39

Freemark M. 609

Frick M. 399

Friesen E. 405

Fuenfer MM. 150

Galeano NF. 67

Gambarara M. 364

Ganapathy V.641

Gärtner J. 488

Garza C. 493

Gauda EB. 683

Geffner MF. 286

Gelband H, 50, 616

Gerstmann DR. 595

Ghishan FK. 173

Gidding CEM. 725

(jiguère R. 67

Gilbert M. 587

Gimotty PA. 330

Giorgi P. 141

Giovanelli $G, 41$

Girard J, 483.587

Glade MJ. 541

Glant TT, 409

Göbel U. 518

Godolphin WJ. 536

Goldberg RN. 478

Golde DW. 286

Goldman AS. 493

Gondek CM. 312

Good RA. 761

Gopal J. 541

Graham TP Jr, 524

Green R. 647

Groothuis DR. 23

Guillet R, 3()?

Gupta D. 41

Gusowski N.16.3

Haddad GG, 306

Hagiwara G. 271

Hakanson DO () 414

Hall J. 449

Hancock REW. 743

Han VKM. 245

Harper TF. 455

Harzer G. 513

Haug M. 513

Hauguel S. 48.

Hazinski rA. 679

Hehre D. 478

I keikkilä J, 445

Henquin J-C. 45

Herrera E. 6

Herson VC. 150

Heyman M. 72

Heymann MA. 389,531

Heymann MH, 350

Hill D). 245

Hill HR. 375

Hintz RI. 188

Hjalmarson $(0,58$

Hofmann AF, 257

Ho I. 312

Holthofer H. 504

Hoppel CI. 312

Horton WA, 324

Hrbek A. 62
Hsu Y'-P, 708

Huang M. 360

Hubner $C .488$

Hughes DM. 83

Hu \. 442

Indo Y, 627

Ingardia $C \mathrm{~J}, 150$

Inzana IJ. 650

Isracis FED, 405

Isracls LG, 405

Ito R. 573

Iwamoto HS. 6.34. 557

Jacob M. 518

Jacobs $\mathrm{HC}, 730$

Jacoby JZ III, 545

Jansen AH, 405

Jansons RA. 92

Jarrells MC. 736

Jeng HJ, 306

Job JC. 39

Johengen M. 55

Johnson I ( . 683

Jose PA, 465

Kalenga M. 45

Kamata K, 201, 282

Kande J. 483

Kaplan SA. 286

Kaplan SI.. 6.50

Karlsson K. 62

Kaufman B. 545

Keller MA. 277

Kelly I)H. 158

Kelly V, 743

Kennaugh JM, 688

Kennedy KA. 679

Kerr DS. 312

Kilborn KM. 158

Kim U-H. 26?

Kimura A. 184

Kitterman JA, 339

Kjellmer I. 62

Klicgman RM. 360)

Klish WJ, 417

Kobayashi Y, 500

Koga Y, 184

Kohlschütter A. 488

Komar KJ. 509)

Kosch PC. 87

Krause PJ. 150

Kries R1. 513

Krumwiede M. 208

Kuipers IM. 725

Kwiathowski K. 344

I.ai TI. 306

I andau I.I. 83

Langston C. 698

l.anouc $\mathrm{KF}, 312$

Lasunción MA. 6

I atte $1: 703$

I.auder J M, 245

Lawson B, 30?

LeBidois J, 350

l.c ( - CH. 339

L(C) I) 344

Leffler C. 647 
Leibach FH, 641

Lepage G, 67

Leroy $\mathrm{C}, 67$

LeSouëf PN, 83

Leturque A, 483

Levin M, 197

Lewiston NJ, 708, 271

$\mathrm{Li} \mathrm{CH}, 286$

Liggins GC, 335, 339

Li K, 720

Lima DM, 730

Lippe BM, 286

Lister G, 177

Liu C-CF, 292

Lockitch G, 536

Loh BA, 429

Louisot P, 250

Lucas A, 675

Lumbers ER, 355

Lundell BPW, 461

Lusk MM, 312

Lynn WS, 545

Machado MA, 324

Maiure L, 703

Martin A, 250

Martin RJ, 683, 87

Mason EO Jr, 659

Matherne GP, 465

Matsuda I, 627

Mauray F, 531

Mayock DE, 449

McCarthy PT, 513

McClung HJ, 720

McCormack J, 50, 616

McCracken GH Jr, 118

McDougall JG, 11

McGilligan KM, 268

Meadow WL, 509

Mercurio MR, 730

Meschia G, 688

Meslin J-C, 72

Meydrech EF, 292

Miller MJ, 683

Milley JR, 168

Mimouni F, 319

Minski MJ, 651

Mirelman D, 130

Mirro R, 647

Momma K, 567, 573

Moreau C, 72

Moreau G, 177

Morton MJ, 621

Mosier HD Jr, 92

Moss M, 177

Moss RB, 708

Motohara K, 627

Moya FR, 627

Muller DPR, 197

Müller W, 123

Mundlos S, 257

Murphy JD, 693

Murray RD, 720

Nagel RA, 286

Nakamura KT, 465

Nakanishi T, 201, 282

Nathoo SA, 1

Neu J, 330
Nielsen HC, 145

Nishida N, 500

Nishikawa T, 573

Nunes CS, 587

Ogata ES, 432

Ogawa S-i, 282

Oh W, 135

Okuda H, 201

O'Laughlin MP, 667

Olley PM, 422

Olsen KD, 118

Olsson T, 581

Orband-Miller L, 599

Overturf GD, 438

Pakarinen A, 445

Palacin M, 6

Parker CR Jr, 145

Pasternak JF, 23

Patel MS, 312

Paul RI, 432

Pearce WJ, 192

Pearson D, 402

Pégorier J-P, 587

Penny R, 414

Petit A, 72

Phelps DL, 297

Pickoff AS, 50, 616

Pier GB, 743

Pino A, 703

Poelmann-Weesjes G, 655

Post M, 79

Price DA, 402

Puterman ML, 536

Queen KL, 599

Quie PG, 760

Quigley G, 536

Quinton PM, 271

Raia V, 703

Raj JU, 714

Raju T, 16

Ranke MB, 41

Rao MB, 330

Rao VS, 292

Raye JR, 150

Redha R, 497

Reed MD, 360

Reid DL, 621

Reid LM, 693

Reisinger D, 277

Reller MD, 621

Reynolds E, 34

Rhodes JB, 257

Rhodes PG, 292

Richard M, 250

Rieger C, 123

Rieger CHL, 399

Rigatto $\mathrm{H}, 344$

Robillard JE, 465

Rodriguez AL, 277

Roels F, 748

Ro $\beta$ kamp R, 41

Roman C, 531

Rose MC, 545

Rosenbaum AL, 297
Rosenfeld CR, 145

Rosenfeld RG, 188

Rosén $\mathrm{KG}, 62$

Rother U, 123

Rothstein G, 455

Roughley PJ, 409

Roy CC, 67

Rudinsky BF, 509

Rudolph AM, 634, 389, 557

Sabetta G, 364

Said HM, 497

Sakane Y, 500

Sandberg K, 58

Santulli TV Jr, 286

Saunders JM, 743

Schadewaldt P, 591

Schellenberg J-C, 335, 339

Scott PH, 651

Segal S, 605

Sekiguchi M, 201

Shalet SM, 402

Shannon DC, 158

Shaul PW, 319

Shaw M, 21

Shearer M, 513

Sheps S, 536

Shine P, 1

Sicard RE, 552

Sidi D, 389

Silano V, 703

Singh VK, 536

Sinniah D, 184

Sjöqvist B-A, 581

Slotkin TA, 599

Smith BT, 79

Smith EO, 667

Smith FG, 355

Smith R, 497

Smith WG, 286

Snyder JM, 145

Soifer SJ, 350

Sonesson S-E, 461

Specker BL, 319

Speert DP, 743, 429

Spencer ME, 92

Standaert TA, 449

Starke JR, 698

States B, 605

Stolfi A, 50, 616

Stonestreet BS, 135

Strain AJ, 245

Strates E, 509

Strohl KP, 87

Sugimoto T, 500

Suguihara C, 478

Surtees RAH, 402

Syrogiannopoulos GA, 118

Taanman JW, 394

Takao A, 201, 282, 567, 573

Tarkka M, 445

Teague WG Jr, 714

Teitel D, 634, 389

Teitel DF, 557

Teng C, 688

Terzi C, 41

Thieriot-Prevost G, 39

Thiringer $\mathrm{K}, 62$
Thomas, DW, 268

Thomas MR, 330

Thornburg KL, 62

Tingle AJ, 536

Tiruppathi $\mathrm{C}, 641$

Tosi MF, 659

Towfighi J, 312

Towle AC, 245

Towstoless MK, 11

Trommer BL, 23

Tsang RC, 319

Uhari M, 445

Ulysses B, 720

Underwood LE, 245

Vamecq J, 748

Van den Branden C, 748

Van Eede PH, 708

Van Epps DE, 383

van Fraasen H, 394

Van Leeuwen AM, 708

Vella $G, 422$

Vermylen C, 197

Vici CD, 364

Villafane J, 50, 616

Virojanavat S, 34

Visser GHA, 655

Waffarn F, 595

Wagerle LC, 302

Wahn V, 123

Walker WR, 330

Walther FJ, 725

Ward J, 438

Watchko JF, 449

Weaver LT, 675

Weiner JS, 360

Wendel U, 591

Werlin SL, 34

Werner JC, 552

Wheeler NC, 277

White JG, 208

White RJ, 409

Whitmore WL, 599

Wiedmeier SE, 262

Wildebrand D, 725

Williams RP, 659

Winberg P, 461

Wine JJ, 271

Winter RJ, 2

Wintour EM, 11

Wong F, 536

Woodrum DE, 449

Woo M, 500

Woronick CL, 150

Wright LL, 54

Wu-Wang CY, 330

Xu H, 50, 616

Yamashita F, 184

Yamashita TS, 360

Yoshida I, 184

Yoshino M, 184

Zelikovic I, 163 